\title{
Simplified Method for Session Coordination Using Multi-level QOS Specification and Translation
}

Nobuhiko NISHIO and Hideyuki TOKUDA

Faculty of Environmental Information, Keio University

5322, Endoh, Fujisawa, Kanagawa 252, Japan

Telephone: $+81-466-47-5111$

Facsimille: $+81-466-47-0835$

E-Mail: $\{$ vino,hxt\}@sfc.keio.ac.jp

\begin{abstract}
In multimedia systems, session coordination technique is important to accommodate a new session and re-distribute reclaimed resources when tough continuous media session is exiting. Although end-users do not want to specify the quality of service (QOS) in an exact memory size like in $\mathrm{KB}$ or $\mathrm{KB} / \mathrm{s}$ of bandwidth, such rigid usages are necessary for admission control and coordination. Therefore, we introduce the multi-level quality specification and QOS translation mechanism to put it into the quantitative expression which the system can handle. Nonetheless, session coordination have demanded complicated computation or presented an NP-complete problem. Constraining QOS specification, we invent a simplified session coordination method which is feasible enough to actually implement. This paper reports experiments on QOS translation and multiple session coordination using the Conductor/Performer system on top of Real-Time Mach 3.0.
\end{abstract}

\section{Keywords}

real-time processing, microkernel, middle-ware, QOS control, continuous media, distributed system

\section{INTRODUCTION}

Quality of service (QOS) management is the indispensable feature in multimedia systems. Although several QOS management systems are proposed, we have not yet met the definitive one. Some proposal might be a mere design framework without any working implementation, the others might offer a fancy power of expression but force a complicated and/or even NP-complete 
computation. Here we explain our QOS control middleware which equipped with QOS translation mechanism and a simple method for dynamic QOS control. This enables us to exchange QOS quantitatively and predictably between sessions and to discard ad hoc system stabilization so far. Furthermore, the session coordination method is simplified by introducing a monotonic constraint into QOS specification. This makes it feasible to actually implement.

In the next section we survey related work, then we introduce our QOS specification in section 3, QOS translation framework in section 4 and our simplified session coordination technique for dynamic resource re-distribution in section 5. Our system is based on Real-Time Mach 3.0 microkernel and the middleware called Conductor/Performer system(Keio-MMP Project WWW 1996, Nishio et al. 1994), which is briefly explained in section 4. Section 6 reports experiments on multimedia session coordination using actual resource usage measurements, and then section 7 offers some concluding remarks.

\section{RELATED WORK}

In resource management for QOS control, Microsoft's Rialto(Jones et al. 1995) presented a QOS reservation framework for multiple types of system resources. There, a resource planner is centered to manage multiple sessions resource coordination. However, any results of actually running system were not presented, and no refer to any QOS translation concept we are interested in.

Although Kawachiya at TRL(Kawachiya et al. 1995) presented an actual timing result of QOS controlling, it only refers to a processor cycle resource.

Tokuda's research(Tokuda et al. 1993) tries to manage multiple multimedia sessions. This control mechanism is active and each thread applies it to itself. This self-stabilization method might not balance resource reclaiming among sessions. Since ordinal multimedia session states would change discretely, a mere simple up-and-down of video frame-rate might damage the overall performance. Especially, a decision of when and how much a session should be upgraded is a hard problem. If failed, not only system instability but also overall system overload might happen.

\section{QOS SPECIFICATION}

\subsection{Characteristics}

QOS specification should be used for predictable multimedia systems. This is utilized for admission control at the session creation, resource reservation and resource re-distribution at the dynamic QOS control. This specification is characterized as the following features: 
- QOS specification is usually discrete and ranged,

- QOS specification has some dimensions,

- objects which are specified have a hierarchy,

- preference between objects is necessary, and

- specification has multiple levels of expression.

First, mainly because of the constraints of devices, QOS specifications are usually discrete and have its upper and lower limits. For example, a video playback at $12 \mathrm{fps}$ is meaningful but playback at $12.3 \mathrm{fps}$ is not. Some device might only have functionality to capture image at $160 \times 120$ or $320 \times 240$. This aspect should be regard as not that every specification is discrete, but that we can not always expect continuity in specifying QOS.

The next aspect refers to QOS dimensions. In our system, it is expressed in two-dimension: temporal quality and spatial quality. Temporal quality concerns video's frame rate or audio's sampling rate, while spatial quality expresses video image size, color-depth bit length, or audio quantization bit length. In future, we plan to add timing accuracy quality, i.e. presentation delay, jitter bound, inter-stream synchronization etc.

The third aspect of objects hierarchy means that a user specify a movie session as a certain quality and the movie session consists of video and audio stream. The video stream might consist of a storage access component, data transport component, compression/ decompression component and viewing component. It is important to clarify which object is specified by its quality.

As the fourth aspect, inter-object constraints of user's preference is necessary. For instance, some user may want to prioritize audio stream more than video stream. Some may regard the session which has the front-most window should be most prioritized. The upper-most component of a data stream would dominate the constraints of the other components in the same stream.

The last aspect of multiple-level expression is explained in the next section.

\subsection{Multi-level QOS Specification}

In multimedia systems, QOS specifications are important for admission control and dynamic resource re-distribution. The nature of such specifications has multiple levels of expression. End-users do not want to know details of system resources like CPU, memory, network bandwidth, etc. They may want to specify a movie playback quality in an abstract way like good/fair/poor. For middleware, it has to analyze the specifications of selected movie file or video camera devices and should use more rigid expressions like 320x240 pixel image, 15 frames per second. On the other hand, systems which have responsibility for admission control, resource reservation and dynamic re-distribution, should obtain quantitative information of resource usage for the movie play- 
Table 1 Three-level QOS characteristics

\begin{tabular}{|c|c|c|c|}
\hline & Expression & User & Example \\
\hline ULQ & abstract & end-user & good/fair/poor \\
\hline MLQ & $\mathrm{H} / \mathrm{W}$ independent & middleware & $24 \mathrm{fps}, 22 \mathrm{kHz}$ \\
\hline SLQ & quantitative & kernel etc. & $18 \%, 20 \mathrm{MB} / \mathrm{s}$ \\
\hline
\end{tabular}

back. They need specific physical memory size or network bandwidth, the usage values of which should change from platform to platform.

We introduce three-level expression of QOS specification. They are UserLevel QOS (ULQ), Middleware-Level QOS (MLQ) and System-Level QOS (SLQ). ULQ is introduced for the end-users, which is expressed in an abstract way like good/fair/poor. MLQ is for middleware-level software for continuous media processing. MLQ has more concrete expression of the QOS than ULQ, for example, $15 \mathrm{fps}, 16$-bit color, $44.1 \mathrm{kHz}$ stereo sampling etc. MLQ should be expressed in hardware platform independent descriptions. The difference between ULQ and MLQ is that MLQ determines an unique QOS of the session, but ULQ does not. Translation between ULQ and MLQ would be usually done in the middleware and/or library linked to an application program.

SLQ is used by kernel, resource manager, admission controller and session coordinator. They should be expressed in rigid expressions like physical memory size and network bandwidth. SLQ contains hardware platform dependent information. They are used for admission control and dynamic resource redistribution in the session coordination. Translation between MLQ and SLQ is usually done by middleware (session server, resource manager etc.) or kernel.

Table 1 shows the characteristics of those three specifications.

\subsection{QOS Path}

Assigning a single QOS specification for a session leads the system to less reactive and less flexible situation. In order to perform dynamic QOS control or resource re-distribution efficiently, multiple QOS states per session should be nominated. Therefore, we introduce a concept of QOS path, which bundles user-admissible QOS states. Every QOS state is totally ordered to form user's preference. Usually the top-most state has the maximum QOS and the bottom one has as low QOS as possible. QOS path is an abstraction of a ordered set of QOS possible states in the user-admissible range. 


\section{QOS TRANSLATION}

In this section, a brief explanation of our middleware is presented first, then the QOS translation mechanism for it is explained.

\subsection{Middleware Architecture}

Before explaining QOS translation mechanism, we must present a brief overview of our software platform. Our middleware is called Conductor/Performer architecture, which is a set of system servers and libraries developed on top of Real-Time Mach 3.0 microkernel.

Conductor is responsible for session management, QOS/resource management and media synchronization. Performers are dedicated in media specific processing; i.e. a file service performer accesses media data directly to/from storage devices, a display performer fetches image data and draws it to a window. User requests conductor for session creation, then conductor negotiates with applicable performers and reserves proper resources. Once a multimedia session is established, a real-time thread is generated in conductor and sends IPC's to performers in order to synchronize their activities. All data transfers through the shared memory buffer (called Cyclic Shared Buffer; CSB) among conductor and performers.

A conductor session consists of one or multiple streams. A stream represents a flow of a single type of medium which does not branch. For example, a movie session consists of a video stream and an audio stream. Each stream is an abstraction of a series of performers. A video stream which displays video frames from a disk storage into a window would be a series of a file performer's session and a display performer's session.

Conductor may delegate the actual system resource reservation and/or enforcement to appropriate entities; processor reservation for microkernel(Mercer et al. 1994) or external dedicated servers(Kawachiya et al. 1995) and storage/network bandwidth for storage/network performers(Kihara et al. 1993).

\subsection{QOS Translation Mechanism}

In the first place, an application program makes a user to select the User-Level QOS (ULQ) for a session via GUI or some other interface. Our toolkit library helps users to include the following things:

- priority of the session among the existing sessions,

- priorities of streams in the session,

- priorities of QOS dimensions in the stream, and

- admissible QOS range of each stream 
First three items are specification between objects(sessions, streams and QOS dimensions). Especially, session priority is the most important, since this is used in a victim selection algorithm for session coordination. Stream precedence specifies which stream is more important for the user, for example, video or audio. These priority may change dynamically. We assume the following cases of priority change:

user's command is issued,
the newly created session should be prioritized the most and
- some window manager which detects the front-most window at that point

The fourth one is given in an abstract way. Our system uses such an expression as max, medium, any. Max means the user satisfies only with the maximum QOS. In this case, QOS path becomes only a single and maximum QOS state. Medium means a user admits the QOS degradation by medium. Any means that he/she does not complain at any QOS.

Since users, of course, specify what a source of stream is (i.e. video camera, a name of movie file etc.), the conductor can know the maximum QOS by asking the source performer. Conductor then translates ULQ expression into a more concrete expression called Middle-ware-Level QOS (MLQ). First, it asks the source performer the available QOS ranks for that stream source. For example, it gives a movie file name to the QuickTime file performer and ask it available QOS-ranked sets of image size, color-depth, frames per second. This set of ranks of service is an MLQ expression. Conductor generates a QOS path based on this. This QOS path expresses the maximum and minimum range of stream QOS. This is the first phase QOS translation from ULQ to MLQ.

Then, the QOS path of MLQ is passed to each performer to examine the necessary resource usage performers actually require, since the corresponding performers only know their activities' physical cost. Therefore, it is desirable for performers to provide conductor with (i)multiply-QOS-ranked services as a QOS translation table and (ii) capability to calibrate cost-estimation for different hardware platforms.Our system's QOS translation table is created off-line. Each performer has a service to calibrate the table according to the hardware platform. Among our performer interfaces, the class_name_open_session service call has a passing-argument of MLQ specification and returning-argument of physical resource usage required. This physical resource usage can be called System-Level QOS (SLQ), which is expressed in processor cycles, physical memory size, or storage/network bandwidth. At this time, conductor could complete the second-phase translation from MLQ into SLQ. This enables conductor to admission-control of new session and session coordinate quantitatively. Session would never be created when conductor decides it is impossible to afford such a QOS range. Figure 1 shows how ULQ is translated into SLQ in our system. 


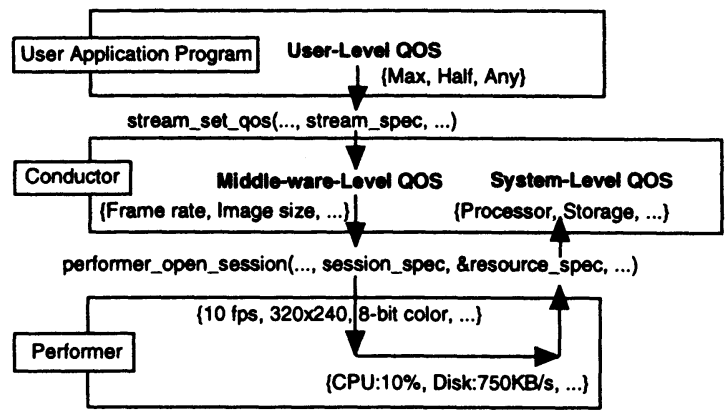

Figure 1 QOS Translation Flow

\section{SIMPLIFIED ALGORITHM FOR SESSION COORDINATION}

Conductor's session coordination which will require resource re-distribution is triggered when:

case 1 A new session creation is requested, case 2 A running session is exiting, case 3 A user requests a QOS change or a performer up-calls the QOS change necessity and

case 4 transient over-load is supposed to occur according to the information collected by a deadline handler of conductor threads.

Then conductor triggers QOS management in the following sequence:

1. victim session selection,

2. calculates deltas of SLQ by QOS-state-transition along QOS path, and examines possibilities of resource re-distribution,

3. executes QOS-state-transition of existing sessions, and

4. reserves resources for a new session, if necessary.

Our victim selection method is quite simple since (i)it is organized only by the session precedence and (ii) we constrain each QOS path must be monotonic. The session precedence is the total order of existing sessions. In our conductor, by default, the most recent session becomes the most important. Users can also specify a session importance explicitly. The monotonic QOS path constraint means that as follows. QOS possible state in SLQ can be a vector of each type of resource usages. These vectors in the QOS path are ordered monotonically decreasing or increasing, i.e. each element is less than or equal to the element in the upper vector in the QOS path. This constraint makes resource usage computation quite simple. 


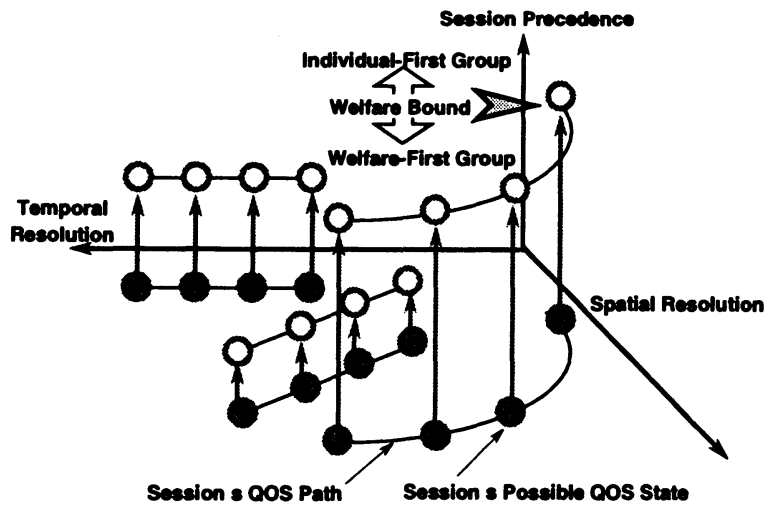

Figure 2 QOS Path and Precedence

Thus victim session can be selected from the session precedence, the lowest session in the sequence. After the victim session's QOS is decreased by onehop, if the reclaimed resource can not accommodate the resource scarcity, the second lowest session is the next victim. The monotonic constraint of QOS paths makes the resource usage computation much easier.

Although there is an alternative policy to decrease the lowest session by two-hop, we do not adopt this, since this policy is suitable for the rather high prioritized sessions. This tends to decrease the number of existing sessions and increase the QOS of them. We call this policy individual-first. We adopt the policy to decrease the QOS of existing sessions and increase the number of them. This policy is called welfare-first.

Therefore, all sessions should be separated into two groups in order to change the policy to reclaim the resource usage. We name the line which separates the sequence of session precedence into two groups welfare bound line; sessions higher than the line are in the individual-first group and other sessions are in the welfare-first group. Sessions in the individual-first group always seek the highest QOS possible using all the available resources in the precedence order. They don't care for sessions lower than themselves. They might force lower sessions to give up to run. Sessions in the welfare-first group seek to preserve or increase the sessions running alive even if their QOS should be minimized. If total number of running sessions gets increased, a higher session might give up to run for lower sessions. Since the number of individualfirst sessions are determined by the hardware potential, actually it would be one or none, in which case all sessions are in the welfare-first group.

Figure 2 depicts the session precedence and QOS paths. 


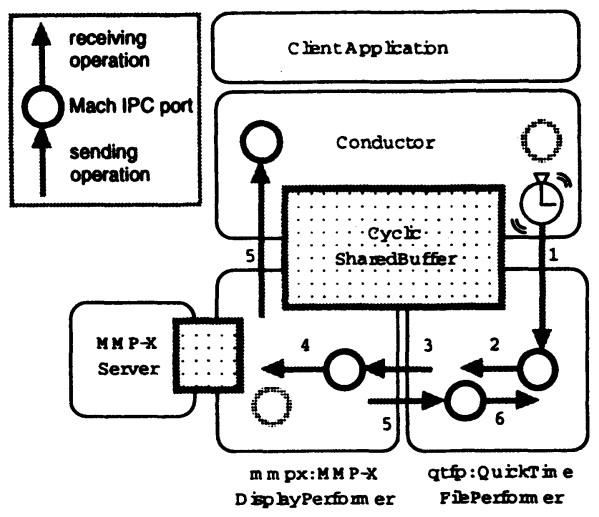

Figure 3 Experiment System

\section{MULTIMEDIA SESSION COORDINATION EXPERIMENT}

We used a hardware platform of Toshiba's Pentium $90 \mathrm{MHz}$ sub-note machine with $16 \mathrm{MB}$ memory and C\&T's 65546 graphics chip on VL-bus architecture. We prepare two performers for this experiments: QuickTime File Performer (qtfp) and MMP-X Display Performer(mmpx).

Qtfp assumes the constant rate storage access server such like CRAS(Tezuka et al. 1994), and simulates such services using memory-mapped file. Although this performer consumes large physical memory, this makes its behavior predictable and suitable for such an experiment. Qtfp can parse Apple's QuickTime movie file and clients can fetch an image frame of any time stamp at the same resource cost through our performer interface. For this experiment, we prepare QuickTime files each has a video track of 50 frames of 8-bit color images but differently sized at $160 \times 120$ and 320x240. Qtfp can be enforced to limit its data transfer rate as client specifies using periodic thread or processor reservation mechanism. Figure 3 shows the system overview.

Mmpx is a performer which we enhanced the $\mathrm{X}$ server from XFree86 v.3.1.2. We made a Mach-IPC port for real-time services besides normal socketinterfaced X services(Tada 1994). Since this service port is always prioritized to normal socket port, we can guarantee $\mathrm{X}$ server's predictable behaviors. In addition to this port, data transfer is done through a shared memory, which completely eliminates image copying and realizes quick image drawing.

All time measurements are done by fetching Pentium's free-running cycle count register, calibrated with its processor cycle.

Our experiment uses three sessions playing QuickTime file into an $\mathrm{X}$ window at the User-Level QOS given in table 2. These QOS specifications are translated into Middle-ware-Level QOS expressions shown in table 3. Session $\mathrm{C}$ is specified as temporal resolution is more important than spatial one.

In our platform, qtfp performer hardly consumes processor cycle, and mmpx 
Table 2 Session Specification: User-Level QOS Expression

\begin{tabular}{lcc}
\hline & Temporal Res. & Spatial Res. \\
\hline Session A & Any & Max \\
\hline Session B & Max & Any \\
\hline Session C & Medium & Medium \\
\hline
\end{tabular}

Table 3 Session Specification: Middle-ware-Level QOS Expression

\begin{tabular}{|c|c|c|}
\hline & Frame Rate & Image Size \\
\hline Session A & $1,2,3,5,6,10,15,30$ & $320 \times 240$ \\
\hline Session B & 30 & $160 \times 120$,magnified, $320 \times 240$ \\
\hline Session C & 15,30 & magnified, $320 \times 240$ \\
\hline
\end{tabular}

performer consumes 1,666 micro seconds per 160x120 image drawing. Other processing like IPC overhead in the conductor costs 638 micro seconds per frame. We limit the maximum storage bandwidth of qtfp by $2,500 \mathrm{~KB} / \mathrm{s}$.

In our conductor setting, only the top-most session belongs to the individualfirst group. Maximum QOS of both Session A, B and C consumes processor cycle $21.9 \%$ and storage bandwidth $2,250 \mathrm{~KB} / \mathrm{s}$. Both resources are affordable for a single session. If we request to create and start session $\mathrm{A}, \mathrm{B}$ and then C, A starts with the maximum QOS. When B starts, since B is the more important than $\mathbf{A}$ and storage bandwidth is not enough, so $\mathbf{A}$ must cut its frame rate to $3 \mathrm{fps}$. When $\mathrm{C}$ starts, the $\max \mathrm{QOS}$ of $\mathrm{C}$ forces to give up to last $B$ but $A$ remains at 3 fps quality.

If we eliminate the individual-first group, nothing changes before $\mathrm{C}$ starts. When $\mathrm{C}$ starts, if $\mathrm{C}$ ran at the maximum, $\mathrm{B}$ must quit to make $\mathrm{C}$ run. Therefore, $\mathrm{C}$ cut its frame rate to $15 \mathrm{fps}$, and $\mathrm{B}$ could continue to run by shrinking storage bandwidth to $562.5 \mathrm{~KB} / \mathrm{s}$, and A could run at $10 \mathrm{fps}$.

Mmpx can magnify the $160 \times 120$ image to $320 \times 240$ by coping, but this consumes processor cycle heavily. In our system this software magnification costs 26 milliseconds per a frame. Therefore, the conductor could not utilize this for $B$ in the all welfare-most situation.

Although it is desirable to have a choice of software image magnification, for our resource re-distribution algorithm, such a choice that upgrades processor resource but degrades storage bandwidth violates our constraint. 
Table 4 Session Specification: System-Level Expression

\begin{tabular}{ccccc}
\hline & \multicolumn{2}{c}{ Processor Cyc. } & \multicolumn{2}{c}{ Storage Bandwidth } \\
\hline Frame Rate & $320 \times 240$ & $160 \times 120$ & $320 \times 240$ & $160 \times 120$ \\
\hline $30 \mathrm{fps}$ & $21.9 \%$ & $6.9 \%$ & $2,250 \mathrm{~KB} / \mathrm{s}$ & $562.5 \mathrm{~KB} / \mathrm{s}$ \\
\hline $15 \mathrm{fps}$ & $11.0 \%$ & $3.5 \%$ & $1,125 \mathrm{~KB} / \mathrm{s}$ & $281.25 \mathrm{~KB} / \mathrm{s}$ \\
\hline $10 \mathrm{fps}$ & $7.3 \%$ & $2.3 \%$ & $750 \mathrm{~KB} / \mathrm{s}$ & $187.5 \mathrm{~KB} / \mathrm{s}$ \\
\hline $6 \mathrm{fps}$ & $4.4 \%$ & $1.4 \%$ & $450 \mathrm{~KB} / \mathrm{s}$ & $112.5 \mathrm{~KB} / \mathrm{s}$ \\
\hline $5 \mathrm{fps}$ & $3.7 \%$ & $1.2 \%$ & $375 \mathrm{~KB} / \mathrm{s}$ & $93.75 \mathrm{~KB} / \mathrm{s}$ \\
\hline $3 \mathrm{fps}$ & $2.2 \%$ & $0.7 \%$ & $225 \mathrm{~KB} / \mathrm{s}$ & $56.25 \mathrm{~KB} / \mathrm{s}$ \\
\hline $2 \mathrm{fps}$ & $1.5 \%$ & $0.5 \%$ & $150 \mathrm{~KB} / \mathrm{s}$ & $37.5 \mathrm{~KB} / \mathrm{s}$ \\
\hline $1 \mathrm{fps}$ & $0.7 \%$ & $0.2 \%$ & $75 \mathrm{~KB} / \mathrm{s}$ & $18.75 \mathrm{~KB} / \mathrm{s}$ \\
\hline
\end{tabular}

\section{CONCLUSION}

For multimedia session coordination it is indispensable to admission control based on quantitative resource management. This assumes that each component of the system must be conscious to its quantitative service cost. Besides, resource reservation and/or enforcement mechanism are also necessary. Our investigation aims such a predictable environment in order to rigidly coordinate multimedia sessions. We introduced (i)a QOS translation mechanism and (ii)a simplified session coordination algorithm which enable to re-distribute system resources among sessions for more stable and predictable session coordination. This paper gave an experiment on the QOS translation using our middleware named Conductor/Performer system and presented an rigid coordination for multiple-graded movie playing sessions.

\section{ACKNOWLEDGMENTS}

The authors would like to thank the members of the MKng project for their help and advice.

\section{REFERENCES}

Keio-MMP Project (1996) World-Wide Web Home Page, URL: <http://wwn.mmp.sfc.keio.ac.jp/>.

Nishio, N. Tada, S. and Tokuda H. (1994) Conductor-Performer: A Middle 
Ware Architecture for Continuous Media Applications. Proc. 1st Intl. Workshop on Real-Time Computing Systems and Applications, 122-31.

Jones, M. B. Leach, P. J. Draves, R. P. and J. S. Barrera, J. S. III. (1995)

Support for User-Centric Modular Real-Time Resource Management in the Rialto Operating System, Proc. of the 5th Int. Workshop on Network and Operating Systems Support for Digital Audio and Video, 55-65.

Kawachiya, K. Ogata, M. Nishio, N. and Tokuda, H. (1995) Evaluation of QOS-Control Servers on Real-Time Mach. Proc. of the 5th Int. Workshop on Network and Operating Systems Support for Digital Audio and Video, 123-6.

Tokuda, H. and Kitayama, T. (1993) Dynamic QOS Control based on RealTime Mach. Proc. of the 4th Int. Workshop on Network and Operating Systems Support for Digital Audio and Video, 113-22.

C. W. Mercer, C. W. Savage, S. and Tokuda, H. (1994) Processor Capacity Reserves: Operating System Support for Multimedia Applications.

Proc. 1st Intl. Conf. on Multimedia Computing and Systems, 90-9.

Kihara, S. Onoe, Y. Mitsuzawa, A. Moriai, S. Nambu, A. and Tokuda, H. (1993) An Implementation of ST-II Protocol as a User-Level Server on Real-Time Mach. The 4th Int. Workshop on Network and Operating Systems Support for Digital Audio and Video.

Tezuka, H. and Nakajima, T. (1994) Design and Implementation of Continuous Media Storage System on Real-Time Mach. JAIST Research Report, IS-RR-94-15S.

Tada, S. (1994) Real-time extension of X window system for continuous media Applications. Proc. of First International Workshop on Real-Time Computing Systems and Applications.

\section{BIOGRAPHY}

\section{Nobuhiko Nishio}

Nishio is an Instructor in the Faculty of Environmental Information at Keio University and received BS degree in engineering and MS degree in science from University of Tokyo.

His research interests include distributed real-time systems, resource reservation and QOS control systems, and mobile systems.

Hideyuki Tokuda, Ph.D. in Computer Science

Prof. Tokuda is a Professor in the Faculty of Environmental Information at Keio University and also an Adjunct Associate Professor in the School of Computer Science at Carnegie Mellon University.

His research interests include distributed real-time systems, multimedia systems, communication protocols, massively parallel/distributed systems, and mobile systems. 